\title{
Adjustment of the dual frequency altimeter low subsection backscatter coefficient statistical model
}

\begin{abstract}
The $\mathrm{Ku}$ band and $\mathrm{C}$ band backscatter coefficient statistical relationship plays an important role in dual frequency altimeter wind speed retrieval when it is rain. In order to improve the precision of wind speed retrieval, it is very important to put forward a proper statistical relationship. Jiang's relationship has higher precision than Quartly's and Yang's relationship between 12dB and 26dB. But if C band backscatter coefficient is $12 \mathrm{~dB}$, the wind speed is $27.3683 \mathrm{~m} / \mathrm{s}$, so if the wind speed is larger than $27.3683 \mathrm{~m} / \mathrm{s}$, Jiang's relationship can't work. High wind speed condition is one of the most important sea phenomena, so we eagerly need to build a new relationship that can apply to the entire sea phenomenon. This paper is based on the specular scattering theory, analyses the low backscatter coefficient subsection's relationship, put forward a method that if $\mathrm{C}$ band backscatter coefficient is between $12 \mathrm{~dB}$ and $26 \mathrm{~dB}$, Jiang's relationship is used, if $\mathrm{C}$ band backscatter coefficient is smaller than $12 \mathrm{~dB}$, Yang's relationship is used. This relationship redounds to improve wind speed retrieval when it is rain.
\end{abstract}

Keywords: dual frequency altimeter, statistical model, backscatter coefficient, low subsection
Volume 3 Issue 3 - 2019

\author{
Zhuhui Jiang, ${ }^{1,2,3}$ Wei Zhang ${ }^{4,5}$ \\ 'State Key Laboratory of Geo-information Engineering, China \\ ${ }^{2}$ International Space University, France \\ ${ }^{3}$ Institute of Atmospheric Physics, Chinese Academy of Sciences, \\ LAGEO, China \\ ${ }^{4}$ Chinese satellite maritime tracking and controlling department, \\ China \\ ${ }^{5}$ College of Meteorology and Oceanography, National University \\ of Defense Technology, China
}

Correspondence: Zhuhui Jiang, State Key Laboratory
of Geo-information Engineering, Xi'an 7 10054, China, Tel
0033766146906 , Email Zhuhui.jiang@community.isunet.edu

Received: July 05, 2019 | Published: July 19, 2019
Chinese classification code: $84.40 . \mathrm{Xb}, 92.60 . \mathrm{Gn}$

\section{Introduction}

NASA and French space agency launched Topex/Poseidon in 1992, and Topex is the first dual-frequency radar altimeter $(\mathrm{Ku}$ band $13.6 \mathrm{GHz}$ and $\mathrm{C}$ band $5.3 \mathrm{GHz}$ ). The initial thought of the equipment of $\mathrm{C}$ band payload is to retrieve the total electron content. There is substantial progress for the altimeter sea surface wind speed error reduction caused by rain because the dual-frequency altimeter is available. Quartly et al., point out that the main influence of rain to radar altimeter echo is attenuating the signal; it is different between the attenuation of $\mathrm{C}$ band backscatter coefficient $\sigma_{C}^{o}$ and that of $\mathrm{Ku}$ band backscatter coefficient $\sigma_{K u}^{o}$ in the rain. They established a statistical relationship model between $\sigma_{C}^{o}$ and $\sigma_{K u}^{o}$ under the assumption of no rain, open sea, no sea ice and small signal deviation angle. The literature ${ }^{2-5}$ also established a statistical model and carried out relevant research of decrease rain influence. The precipitation capacity of $10 \mathrm{~mm} / \mathrm{h}$ causes the signal attenuation of about $4.5 \mathrm{~dB}$ for $\mathrm{Ku}$ band and about 0.26 for $\mathrm{C}$ band. If the backscatter error is $0.1 \mathrm{~dB}$, the retrieved wind speed error is about $0.3 \mathrm{~m} / \mathrm{s}$ in moderate wind, so the backscatter coefficient statistical model is very important for rain influence reduction. The amount of trial data is too small for the backscatter coefficient statistical model of the literature. ${ }^{1}$ The statistical relationship model established in the literature ${ }^{5}$ is a piecewise linear function, which is disconnected at the link points. Jiang et al., ${ }^{6}$ presented a new backscatter coefficient statistical model with 7 and a half years Jason-1 data by a polynomial fitting method to solve above problems, but the available range of $\mathrm{C}$ band backscatter coefficient is only $12 \mathrm{~dB}$ to $26 \mathrm{~dB}$. C band backscatter coefficient of $12 \mathrm{~dB}$ corresponds to the sea surface wind speed of about $27 \mathrm{~m} / \mathrm{s}$ by calculation, which means that if the sea surface wind speed is higher than $27 \mathrm{~m} / \mathrm{s}$, the model of literature ${ }^{5}$ lose effectiveness. However, high wind speed and severe sea situation often cause tremendous disasters, so it is necessary to research low subsection backscatter coefficient statistical relationship.

In this paper, the low subsection backscatter coefficient statistical relationship of $\mathrm{C}$ band and $\mathrm{Ku}$ band is analyzed basing on the specular scattering theory in section 2 , the accuracies of different methods are evaluated in section 3 , and an adjusted method is put forward that if $\mathrm{C}$ band backscatter coefficient is in the range of $12 \mathrm{~dB}$ to $26 \mathrm{~dB}$, the method of literature ${ }^{6}$ is available, and if $\mathrm{C}$ band backscatter coefficient is below $12 \mathrm{~dB}$, the method of literature ${ }^{5}$ is available. The adjusted method can improve sea surface wind speed accuracy under rainfall conditions.

The basic theory of wind speed inversion of dual-frequency altimeter under rainfall condition

The influence of rainfall on the radar signal can be obtained by the function proposed by Marshall Palmer

$$
k=a R^{b}
$$

where $k$ refers to the absorption coefficient, $R$ is the precipitation capacity, $a$ and $b$ are coefficients determined by radar frequency.

The total attenuation of radar signal emission and reception follows the following relation,

$$
A=2 k h=2 h a R^{b}
$$

where $h$ is the height of rainfall.

Therefore, there is the following relationship between rainfall and the attenuation intensity caused by rainfall

$$
R=\left(\frac{A}{2 h a}\right)^{1 / b}
$$


$\mathrm{a}=34.6 \times 10^{-3}, b=1.109$ for $\mathrm{Ku}$ band, $\mathrm{a}=1.06 \times 10^{-3}, b=1.393$ for $\mathrm{C}$ band.

The basic iterative method ${ }^{2,7-10}$ for the inversion of wind speed by dual-frequency altimeter under rainfall condition is

First, estimate whether it is rain according to the backscatter coefficient statistical model of between $\sigma_{C}^{o}$ and $\sigma_{K u}^{o}$. If it is not rain, jump out of iteration;

If it is rain, $\sigma_{K u}^{o}$ is substituted into the statistical model to obtain the ideal backscatter coefficient of $\mathrm{Ku}$ band $\sigma_{K u}^{o^{\prime}}$, and then the signal attenuation of $\mathrm{Ku}$ band is obtained by comparing with it, $A_{K u}=\sigma_{K u}^{o^{\prime}}-\sigma_{K u}^{o}$;

Precipitation capacity $\mathrm{R}$ can be calculated by equation (1). $\mathrm{R}$ is independent of the radar signal, so the corresponding $\mathrm{C}$ band signal attenuation $A_{C}$ can be calculated by equation (1). The signal attenuation of $\mathrm{C}$ band is $\sigma_{C}^{o^{\prime}}=\sigma_{C}^{o}+A_{C}$;

The new signal attenuation of $\mathrm{Ku}$ band $A_{K u}^{\prime}$ can be obtained by substituting $\sigma_{K u}^{o^{\prime}}$ and $\sigma_{K u}^{o^{\prime}}$ into the statistical relationship;

Judge whether $\sigma_{K u}^{o^{\prime}}$ is less than a certain threshold, if it is true, jump out of the iteration, and retrieve the sea surface wind speed by the corrected $\sigma_{K u}^{o^{\prime}}$. If it is false, go to 'c' and continue the iteration.

The precipitation capacity of $10 \mathrm{~mm} / \mathrm{h}$ causes the signal attenuation of about $4.5 \mathrm{~dB}$ for $\mathrm{Ku}$ band and about 0.26 for $\mathrm{C}$ band by equation (1). If the backscatter error is $0.1 \mathrm{~dB}$, the retrieved wind speed error is about $0.3 \mathrm{~m} / \mathrm{s}$ in the moderate wind from the Modified Chelton and Wentz Wind Speed Model Function (MCW), so the statistical model has important effects on wind speed retrieval.

\section{Backscatter coefficient statistical model}

There is a certain correlation between $\sigma_{K u}^{o}$ and $\sigma_{K u}^{o}$ physically, but this correlation cannot be deduced theoretically ${ }^{2}$ which is usually based on empirical fitting.

Quartly et al., ${ }^{1}$ analyzed the influence of precipitation ideally on altimeter wind speed retrieval using Topex data. They suggested that the $\mathrm{C}$ band backscatter coefficient could be used to distinguish rain or not from the different influence on a different frequency. Forwardly, a piecewise statistical model fitting from Topex data from December 1992 to October 1993 (Cycle 11, 12, 21, 22, 29, 30, 39 and 40) is put

Table I Yang ${ }^{5}$ subsection coefficient of her standard relationship forward ignoring conditions that the liquid water content is less than $0.2 \mathrm{~mm}$.

$$
\begin{aligned}
& \sigma_{K u}^{o}=8.99+1.19\left(\sigma_{C}^{o}-13.0\right), \quad \sigma_{C}^{o}<13.0 d B ; \\
& \sigma_{K u}^{o}=8.99+1.52\left(\sigma_{C}^{o}-13.0\right), \quad \sigma_{C}^{o} \in[13.0 d B, 14.0 d B) ; \\
& \sigma_{K u}^{o}=10.51+1.21\left(\sigma_{C}^{o}-14.0\right), \quad \sigma_{C}^{o} \in[14.0 d B, 14.7 d B) ; \\
& \sigma_{K u}^{o}=11.36+0.89\left(\sigma_{C}^{o}-14.7\right), \sigma_{C}^{o} \in[14.7 d B, 15.7 d B) ; \\
& \sigma_{K u}^{o}=12.25+0.72\left(\sigma_{C}^{o}-15.7\right), \sigma_{C}^{o} \in[15.7 d B, 16.2 d B) ; \\
& \sigma_{K u}^{o}=12.61+0.86\left(\sigma_{C}^{o}-16.2\right), \sigma_{C}^{o} \in[16.2 d B, 19.4 d B) ; \\
& \sigma_{K u}^{o}=15.36+0.96\left(\sigma_{C}^{o}-19.4\right), \sigma_{C}^{o} \in[19.4 d B, 21.0 d B) ; \\
& \sigma_{K u}^{o}=16.90+1.07\left(\sigma_{C}^{o}-21.0\right), \sigma_{C}^{o} \in[21.0 d B, 24.2 d B) ; \\
& \sigma_{K u}^{o}=20.31+1.11\left(\sigma_{C}^{o}-24.2\right), \sigma_{C}^{o} \in[24.2 d B, 26.0 d B) ; \\
& \sigma_{K u}^{o}=\text { undefined }, \quad \sigma_{C}^{o} \geq 26.2 d B .
\end{aligned}
$$

Yang $^{5}$ suggested a piecewise statistical model using piecewise linear fitting by Jason-1 data from March 2006 to February 2007 (Cycle 155, 157, 159, 163, 167, 169, 172, 174, 176, 180, 184 and 188) (Table 1). Its expression is

$$
\sigma_{K u}^{o}=f\left[\sigma_{C}^{o}\right]=c_{1} \times \sigma_{C}^{o}+c_{2}
$$

Quartly et al., ${ }^{1}$ and Yang ${ }^{5}$ fitted the statistical model by 80 days and 120 days data respectively, the amount of data is small and unrepresentative. The piecewise linear function link points of Yang ${ }^{5}$ are disconnected.

Jiang et al., ${ }^{6}$ presented a new backscatter coefficient statistical model with 7 and a half years no rain, open sea, no sea ice and small signal deviation angle Jason-1 data by polynomial fitting method to solve above problems, but the available range of $\mathrm{C}$ band backscatter coefficient is only $12 \mathrm{~dB}$ to $26 \mathrm{~dB}$ (Equation 4). The fitting coefficients are listed in Table 2.

$$
\sigma_{K u}^{o}=f\left(\sigma_{C}^{o}\right)=a\left(\sigma_{C}^{o}\right)^{6}+b\left(\sigma_{C}^{o}\right)^{5}+c\left(\sigma_{C}^{o}\right)^{4}+d\left(\sigma_{C}^{o}\right)^{3}+e\left(\sigma_{C}^{o}\right)^{2}+f\left(\sigma_{C}^{o}\right)+g
$$

The above three statistical model standard deviations are listed in Table 3. Jiang et al., ${ }^{6}$ standard deviations are significantly better between $12 \mathrm{~dB}$ to $26 \mathrm{~dB}$ than the standard deviation of Quartly et al., ${ }^{1}$ and Yang. ${ }^{5}$ Only in the range of $12 \mathrm{~dB}$ to $15 \mathrm{~dB}$, the accuracy of Yang ${ }^{5}$ is higher than Jiang et al. ${ }^{6}$, the accuracy of Jiang et al., ${ }^{6}$ are very high in the rest of the range.

\begin{tabular}{lccccc}
\hline$\sigma_{C}^{o}$ range/dB & $c_{1}$ & $\sigma_{C}^{o}$ & $\sigma_{C}^{o}$ range $/ \mathrm{dB}$ & $c_{1}$ & $c_{2}$ \\
\hline $16.2 \leq \sigma_{C}^{o}<19.4$ & 1.202 & -7.086 & $16.2 \leq \sigma_{C}^{o}<19.4$ & 0.894 & -1.829 \\
$13.0 \leq \sigma_{C}^{o}<14.0$ & 1.471 & -10.602 & $19.4 \leq \sigma_{C}^{o}<21.0$ & 0.916 & -2.23 \\
$14.0 \leq \sigma_{C}^{o}<14.7$ & 1.527 & -11.357 & $21.0 \leq \sigma_{C}^{o}<24.2$ & 1.026 & -4.498 \\
$14.7 \leq \sigma_{C}^{o}<15.7$ & 1.099 & -5.023 & $24.2 \leq \sigma_{C}^{o}<26.0$ & 0.849 & -0.203 \\
$15.7 \leq \sigma_{C}^{o}<16.2$ & 0.914 & -2.146 & $26.2 \leq \sigma_{C}^{o}$ & 0.099 & 19.334 \\
\hline
\end{tabular}


Table 2 jiang et al., ${ }^{6}$ coefficients

\begin{tabular}{ll}
\hline coefficients & value \\
\hline$a$ & $23.759|600| 70 \mid 60 \mathrm{e}-006$ \\
$b$ & $-2.767 \mid 0569836364 \mathrm{e}-003$ \\
$c$ & $\mid 32.238924476659 \mathrm{e}-003$ \\
$e$ & $-3.3|4388| 6243354$ \\
$e$ & $45.87|360367650|$ \\
$f$ & -330.723722504547 \\
$g$ & $975.195895449 \mid 45$ \\
\hline
\end{tabular}

Table 3 Standard deviation of the three standard relationships

\begin{tabular}{lllll}
\hline standard deviation & $\sigma_{C}^{o} \in[12,26] / \mathrm{dB}$ & $\sigma_{C}^{o} \in[12,15) / \mathrm{dB}$ & $\sigma_{C}^{o} \in[15,20) / \mathrm{dB}$ & $\sigma_{C}^{o} \in[20,23] / \mathrm{dB}$ \\
\hline Jiang et al., & 0.0543 & 0.0606 & 0.0602 & 0.0384 \\
Yang $^{5}$ & 0.1896 & 0.0273 & 0.086 & 0.2634 \\
Quartly et al.,', & 0.3124 & 0.439 & 0.1827 & 0.3843 \\
\hline
\end{tabular}

\section{Adjustment of the backscatter coefficient statistical model}

This section will discuss the shortcomings of the statistical relationship in Jiang et al., ${ }^{6}$ and advance the adjusted methods.

The statistical scatter diagram of Jason-1 radar altimeter from cycle 1 to cycle 279 is shown in Figure 1. The samples selected criterion is no rain, open sea, no sea ice and small signal deviation angle. Because the accuracy of backscatter coefficient values of Jason-1's GDR (Geophysical Data Records) is $0.01 \mathrm{~dB}$, the scatter points are drawn every $0.01 \mathrm{~dB}$ during statistics.

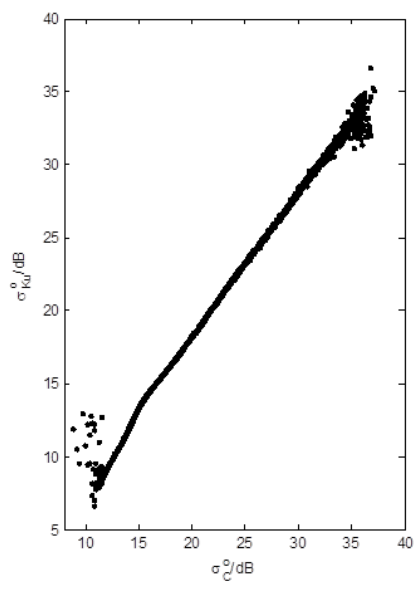

Figure I The $\sigma_{C}^{o}$ and $\sigma_{K u}^{o}$ statistical scatter diagram of Jason-I radar altimeter from cycle I to cycle 279.
The scatter points are relatively concentrated when $\sigma_{C}^{o} \in[12,26)$ , and the correlation of $\mathrm{Ku}$ band and $\mathrm{C}$ band backscatter coefficient is obvious. But the scatter points are relatively divergent when $\sigma_{C}^{o}<12$ $\mathrm{dB}$ and $\sigma_{C}^{o}>26 \mathrm{~dB}$, this is the reason that Jiang et al., ${ }^{6}$ adopted the range of $\sigma_{C}^{o} \in[12,26)$.

Some samples of sea surface wind speed value corresponding to Ku band backscatter coefficients using the method of Jiang et al., ${ }^{11}$ are listed in Table 4.

When the significant wave height is $1 \mathrm{~m}$ and $\sigma_{C}^{o}=23.3 \mathrm{~dB}$, the calculated wind speed value is $0.0084 \mathrm{~m} / \mathrm{s}$. Since the higher the backscatter coefficient of radar altimeter is, the smaller the corresponding wind speed value is, so the condition of $\sigma_{C}^{o}>26 \mathrm{~dB}$ will not be considered. According to equation (4), when $\sigma_{C}^{o}=12 \mathrm{~dB}$, $\sigma_{K u}^{o}=9.0800 \mathrm{~dB}$, the corresponding wind speed value is $27.3683 \mathrm{~m} / \mathrm{s}$, that is to say, when the wind speed calculated by radar altimeter is greater than $27.3683 \mathrm{~m} / \mathrm{s}, \sigma_{C}^{o}$ should be less than $12 \mathrm{~dB}$. High wind speed and severe sea situation often cause tremendous disasters, so it is necessary to research the statistical model when $\sigma_{C}^{o}<12 \mathrm{~dB}$. The $\mathrm{Ku}$ band and $\mathrm{C}$ band statistical model of equation (4) in the range of $\sigma_{C}^{o} \in[8,32]$ is shown in Figure 2. When $\sigma_{C}^{o} \in[8,12]$, it means that $\sigma_{C}^{o}$ decreases if $\sigma_{C}^{o}$ increases which violates the specular scattering theory of radar altimeter. This paper adopts the statistical model of Yang $^{5}$ in the range of $\sigma_{C}^{o} \in[8,12$ ) (The dotted line in figure 2). The reason is that the accuracy of Yang ${ }^{5}$ is the highest when $\sigma_{C}^{o} \in[8,12)$ in Table 3 which meets the specular scattering theory. 
That is, Yang ${ }^{5}$ is adopted when $\sigma_{C}^{o} \in[8,12)$, while Jiang et al., ${ }^{6}$ is adopted when $\sigma_{C}^{o} \in[12,26)$.

Table 4 The correspondence of sea surface wind speed and significant wave height with $\sigma_{K u}^{o}$

\begin{tabular}{lll}
$\sigma_{K u}^{o} / \mathrm{dB}$ & Wind speed $/ \mathrm{m} \cdot \mathrm{s}^{-1}$ & Significant wave height $/ \mathrm{m}$ \\
\hline 23.300 & 0.0084 & $\mathrm{I}$ \\
10.2313 & 20 & - \\
9.0800 & 27.3683 & - \\
8.6688 & 30 & - \\
7.1063 & 40 & - \\
5.5438 & 50 & - \\
\hline
\end{tabular}

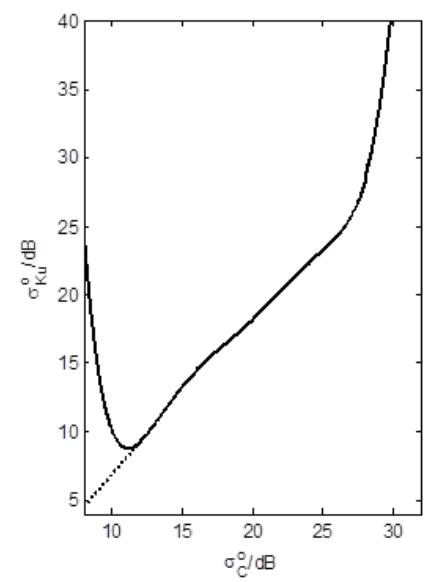

Figure 2 The backscatter coefficient statistical model in the range of $\dot{o}_{\mathrm{C}}^{\mathrm{o}} \in[8,32)$.

\section{Conclusions}

The backscatter coefficient statistical model plays an important role in the sea surface wind speed retrieval using dual frequency altimeter. This paper suggests an adjustment of low subsection backscatter coefficient statistical relationship, that is, Yang ${ }^{5}$ is adopted when $\sigma_{C}^{o} \in[8,15)$, while Jiang et al., ${ }^{6}$ is adopted when $\sigma_{C}^{o} \in[15,26)$. The adjustment will improve the accuracy of the statistical model, and have a positive affect for high wind speed retrieval. Work in this paper and Jiang et al., ${ }^{6}$ only consider the statistical relationship between $\sigma_{C}^{o}$ and $\sigma_{K u}^{o}$ without any physical mechanism research, and is short of in situ data verification. Further work will consider these aspects.

\section{Funding details}

Project Supported by Foundation of State Key Laboratory of Geo-information Engineering (SKLGIE2018-ZZ-8), the Postdoctoral Science Foundation of China(2017M610971).

\section{Acknowledgments}

None.

\section{Conflicts of interest}

Authors declare that there is no conflict of interest.

\section{References}

1. Quartly GD, Guymer TH, Srokosz MA. The effects of rain on Topex radar altimeter data. J Atmos Oceanic Tech. 1996;13:1209-1229.

2. Quilfen Y, Tournadre J, Chapron B. Altimeter dual-frequency observations of surface winds, waves, and rain rate in tropical cyclone Isabel. $J$ Geophys Res. 2006;111(C1):C01004.

3. Chen G, Chapron B, Ezraty R, et al. A dual-frequency approach for retrieving sea surface wind speed from Topex altimetry. $J$ Geophys Res. 2002;107(C12):3226-3235.

4. Yang L, Lin M, Zou J, et al. Improving the wind and wave estimation of dual-frequency altimeter JASON1 in Typhoon Shanshan and considering the rain effects. Acta Oceanologica Sinica. 2008;27(5):49-62.

5. Yang Le. Study on satellite radar altimeter retrieval algorithms over coastal seas and under heigh sea state events. Nanjing: Nanjing University of Science and Technology. Ph. D. Dissertion; 2009 (in Chinese).

6. Jiang Z, Huang S, Liu B. Standard relationship adjustment of dual frequency altimeter backscatter coefficient. Journal of PLA University of Science and Technology(Natural Science Edition). 2011;12(5):555-558.

7. Jiang Z, Huang S, Du Hua-dong, et al. A new approach to adjust sea surface wind using altimeter wind data by regularization and variational method. Acta Phys Sin. 2010;59(12):646-655.

8. Jiang Z, Huang S, Liu G, et al. Research on the development of surface wind speed retrieval from satellite radar altimeter. Marine Science Bulletin. 2011;30(5):588-594.

9. Yang L, Zou J, Lin M, et al. Method to correct both foam and rain effects on dual frequency altimeter Jason1 wind measurements in typhoon Shanshan. In proceedings of SPIE, Remote sensing of the ocean, sea ice, and large water regions; 2008 .

10. Jiang $\mathrm{Z}, \mathrm{Li} \mathrm{Y}, \mathrm{Yu} F$, et al. A damped Newton variational inversion method for SAR wind retrieval. J Geophys Res Atmos. 2017;122(2):823-845.

11. Jiang Z, Huang S, Guo H, et al. Research on the combination of VandemarkChapron algorithm and Young algorithm to retrieve the altimeter sea surface wind speed. Marine Science Bulletin. 2011;30(6):82-85. 